Bull. Korean Math. Soc. 50 (2013), No. 3, pp. 833-837

http://dx.doi.org/10.4134/BKMS.2013.50.3.833

\title{
A NOTE ON THE GENERALIZED MYERS THEOREM FOR FINSLER MANIFOLDS
}

\author{
BING-YE Wu
}

ABSTRACT. In this note we establish a generalized Myers theorem under line integral curvature bound for Finsler manifolds.

\section{Introduction}

The celebrated Myers theorem in global Riemannian geometry says that if a Riemannian manifold $M$ satisfies $\operatorname{Ric}(v) \geq(n-1) a>0$ for all unit vector $v$, then $M$ is compact and

$$
\operatorname{diam}(M) \leq \frac{\pi}{\sqrt{a}}
$$

There are many generalizations of Myers theorem (see e.g., $[2,3,7]$ ). In [7] the author proved the following result.

Theorem 1.1. Let $(M, g)$ be an n-dimensional complete Riemannian manifold. Then for any $\delta>0, a>0$, there exists $\epsilon=\epsilon(n, a, \delta)$ satisfying the following. If for any $p \in M$ and each minimal geodesic $\gamma$ emanating from $p$, the Ricci curvature satisfies

$$
\int_{\gamma} \max \left\{(n-1) a-\operatorname{Ric}\left(\gamma^{\prime}(t)\right), 0\right\} d t \leq \epsilon(n, a, \delta),
$$

then $M$ is compact with

$$
\operatorname{diam}(M) \leq \frac{\pi}{\sqrt{a}}+\delta
$$

Myers theorem has also been generalized to Finsler manifolds [1]. In this note we shall prove the following result which generalizes Theorem 1.1.

Theorem 1.2. Let $(M, F)$ be an n-dimensional forward complete Finsler manifold. If there is $\Lambda>0$ such that for any $p \in M$ and each minimal geodesic $\gamma$

Received March 9, 2012.

2010 Mathematics Subject Classification. Primary 53C60; Secondary 53B40.

Key words and phrases. Myers theorem, Ricci curvature, Finsler manifold.

The research was supported by the Natural Science Foundation of China (No. 11171139). 
emanating from $p$, the Ricci curvature satisfies

$$
\int_{\gamma} \max \left\{(n-1) a-\operatorname{Ric}\left(\gamma^{\prime}(t)\right), 0\right\} d t \leq \Lambda,
$$

then $M$ is compact with

$$
\operatorname{diam}(M) \leq \frac{\pi}{\sqrt{a}}+\frac{\Lambda}{(n-1) a} .
$$

\section{Finsler geometry}

In this section we briefly recall some fundamental materials of Finsler geometry, and for details one is referred to see $[1,4,5,6]$. Let $(M, F)$ be a Finsler $n$-manifold with Finsler metric $F: T M \rightarrow[0, \infty)$. Let $(x, y)=\left(x^{i}, y^{i}\right)$ be local coordinates on $T M$, and $\pi: T M \backslash 0 \rightarrow M$ the natural projection. Unlike in the Riemannian case, most Finsler quantities are functions of $T M$ rather than $M$. The fundamental tensor $g_{i j}$ is defined by

$$
g_{i j}(x, y):=\frac{1}{2} \frac{\partial^{2} F^{2}(x, y)}{\partial y^{i} \partial y^{j}} .
$$

Let $R_{j k l}{ }^{i}$ be the first Chern curvature tensor, and $R_{i j k l}:=g_{j s} R_{i k l}{ }^{s}$. Write $\mathbf{g}_{y}=g_{i j}(x, y) d x^{i} \otimes d x^{j}, \mathbf{R}_{y}=R_{i j k l}(x, y) d x^{i} \otimes d x^{j} \otimes d x^{k} \otimes d x^{l}$. For a tangent plane $P \subset T_{x} M$, let

$$
\mathbf{K}(P, y)=\mathbf{K}(y ; u):=\frac{\mathbf{R}_{y}(y, u, u, y)}{\mathbf{g}_{y}(y, y) \mathbf{g}_{y}(u, u)-\left[\mathbf{g}_{y}(y, u)\right]^{2}},
$$

where $y, u \in P$ are tangent vectors such that $P=\operatorname{span}\{y, u\}$. We call $\mathbf{K}(P, y)$ the flag curvature of $P$ with flag pole $y$. Let

$$
\mathbf{R i c}(y)=\sum_{i} \mathbf{K}\left(y ; e_{i}\right)
$$

where $\left\{e_{1}, \ldots, e_{n}\right\}$ is a $\mathbf{g}_{y}$-orthogonal basis for the corresponding tangent space. We call $\mathbf{R i c}(y)$ the Ricci curvature of $y$.

Let $V=v^{i} \partial / \partial x^{i}$ be a non-vanishing vector field on an open subset $\mathcal{U} \subset M$. One can introduce a Riemannian metric $\widetilde{g}=\mathbf{g}_{V}$ and a linear connection $\nabla^{V}$ (called Chern connection) on the tangent bundle over $\mathcal{U}$ as follows:

$$
\nabla_{\frac{\partial}{\partial x^{i}}}^{V} \frac{\partial}{\partial x^{j}}:=\Gamma_{i j}^{k}(x, v) \frac{\partial}{\partial x^{k}}
$$

where $\Gamma_{j k}^{i}(x, v)$ are the Chern connection coefficients.

The Legendre transformation $l: T M \rightarrow T^{*} M$ is defined by

$$
l(Y)= \begin{cases}\mathbf{g}_{Y}(Y, \cdot), & Y \neq 0 \\ 0, & Y=0\end{cases}
$$

Now let $f: M \rightarrow \mathbb{R}$ be a smooth function on $M$. The gradient of $f$ is defined by $\nabla f=l^{-1}(d f)$. Thus we have

$$
d f(X)=\mathbf{g}_{\nabla f}(\nabla f, X), \quad X \in T M .
$$


Let $\mathcal{U}=\left\{x \in M:\left.\nabla f\right|_{x} \neq 0\right\}$. We define the Hessian $H(f)$ of $f$ on $\mathcal{U}$ as follows:

$$
H(f)(X, Y):=X Y(f)-\nabla_{X}^{\nabla f} Y(f), \quad \forall X,\left.Y \in T M\right|_{\mathcal{U}} .
$$

It is known that $H(f)$ is symmetric, and it can be rewritten as (see [6])

$$
H(f)(X, Y)=\mathbf{g}_{\nabla f}\left(\nabla_{X}^{\nabla f} \nabla f, Y\right)
$$

It should be noted that the notion of Hessian defined here is different from that in [4]. In that case $H(f)$ is in fact defined by

$$
H(f)(X, X)=X \cdot X \cdot(f)-\nabla_{X}^{X} X(f),
$$

and there is no definition for $H(f)(X, Y)$ if $X \neq Y$. The advantage of our definition is that $H(f)$ is a symmetric bilinear form and we can treat it by using the theory of symmetric matrix. For any fixed $p \in M$ let $r=d_{F}(p, \cdot)$ be the distance function from $p$ induced by Finsler metric $F$, and $(r, \theta)$ the polar coordinates on $M \backslash C(p)$, where $C(p)$ is the cut loci of $p$. The following lemma is crucial to prove Theorem 1.2.

Lemma 2.1. Let $h=h(r, \theta)=$ trace $_{\mathbf{g}_{\nabla r}} H(r)$. Then $\lim _{r \rightarrow+0} h=+\infty$, and

$$
\frac{d h}{d r} \leq-\boldsymbol{R i c}(\nabla r)-\frac{h^{2}}{n-1} .
$$

Proof. Let $E_{1}, \ldots, E_{n-1}, E_{n}=\nabla r$ be the local $\mathbf{g}_{\nabla r}$-orthonormal frame fields on $M \backslash C(p)$. We have the following equality where $r$ is smooth (see (5.1) of $[6])$ :

$$
\frac{d}{d r} \operatorname{trace}_{\mathbf{g}_{\nabla r}} H(r)=-\mathbf{R i c}(\nabla r)-\sum_{i, j}\left(H(r)\left(E_{i}, E_{j}\right)\right)^{2} .
$$

Note that $\nabla r$ is a geodesic field, and thus $H(r)(\nabla r, \cdot)=0$, which together with above equality and Schwartz inequality we clearly have the desired inequality. On the other hand, for sufficiently small $\epsilon$ let $b$ be the upper bound of flag curvature on $B_{p}(\epsilon)$, then by Hessian comparison theorem [6] it follows that

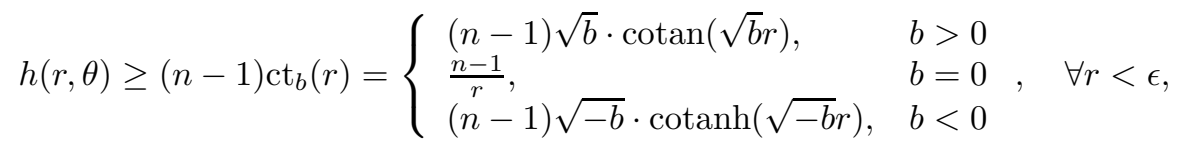

and consequently, $\lim _{r \rightarrow+0} h=+\infty$.

\section{Proof of Theorem 1.2}

Now let us complete the proof of Theorem 1.2. For any fixed $p, q \in M$ let $\gamma:[0, L] \rightarrow M$ be the minimal unit-speeded geodesic from $p$ to $q$ with $L=r(q)=d_{F}(p, q)$. Let $h=h(r, \theta)$ be defined by Lemma 2.1, and consider $f=f(t):=h(\gamma(t))$, then $f$ is smooth on $(0, L)$. By Lemma 2.1 one has

$$
f^{\prime}(t) \leq-\operatorname{Ric}\left(\gamma^{\prime}(t)\right)-\frac{f(t)^{2}}{n-1}
$$


and consequently,

$$
\left(\operatorname{arccot}\left(\frac{f}{(n-1) \sqrt{a}}\right)\right)^{\prime}=\frac{-\frac{1}{(n-1) \sqrt{a}} f^{\prime}}{1+\frac{f^{2}}{(n-1)^{2} a}}
$$

$$
\begin{aligned}
& \geq \frac{\operatorname{Ric}\left(\gamma^{\prime}\right)+\frac{f^{2}}{n-1}}{\left(1+\frac{f^{2}}{(n-1)^{2} a}\right)(n-1) \sqrt{a}} \\
& =\frac{\operatorname{Ric}\left(\gamma^{\prime}\right)-(n-1) a+(n-1) a\left(1+\frac{f^{2}}{(n-1)^{2} a}\right)}{\left(1+\frac{f^{2}}{(n-1)^{2} a}\right)(n-1) \sqrt{a}} \\
& \geq-\frac{1}{(n-1) \sqrt{a}} \max \left\{(n-1) a-\operatorname{Ric}\left(\gamma^{\prime}\right), 0\right\}+\sqrt{a} .
\end{aligned}
$$

For any small $\epsilon>0$ integrating $(1)$ on $(\epsilon, L-\epsilon)$ we get

$$
\begin{aligned}
& \pi-\operatorname{arccot}\left(\frac{f(\epsilon)}{(n-1) \sqrt{a}}\right) \\
(2)> & \operatorname{arccot}\left(\frac{f(L-\epsilon)}{(n-1) \sqrt{a}}\right)-\operatorname{arccot}\left(\frac{f(\epsilon)}{(n-1) \sqrt{a}}\right) \\
\geq & -\frac{1}{(n-1) \sqrt{a}} \int_{\epsilon}^{L-\epsilon} \max \left\{(n-1) a-\operatorname{Ric}\left(\gamma^{\prime}(t)\right), 0\right\} d t+(L-2 \epsilon) \sqrt{a} .
\end{aligned}
$$

On the other hand, $\lim _{t \rightarrow+0} f(t)=+\infty$ by Lemma 2.1 , and thus

$$
\lim _{t \rightarrow+0} \operatorname{arccot}\left(\frac{f(t)}{(n-1) \sqrt{a}}\right)=0 .
$$

Now let $\epsilon \rightarrow+0$ in (2) it follows that

$$
\begin{aligned}
\pi & \geq-\frac{1}{(n-1) \sqrt{a}} \int_{0}^{L} \max \left\{(n-1) a-\operatorname{Ric}\left(\gamma^{\prime}(t)\right), 0\right\} d t+L \sqrt{a} \\
& \geq-\frac{\Lambda}{(n-1) \sqrt{a}}+L \sqrt{a},
\end{aligned}
$$

and consequently,

So we complete the proof.

$$
L \leq \frac{\pi}{\sqrt{a}}+\frac{\Lambda}{(n-1) a} .
$$

\section{References}

[1] D. Bao, S. S. Chern, and Z. Shen, An Introduction to Riemann-Finsler Ggeometry, GTM 200, Springer-Verlag, 2000.

[2] C. Chicone and P. Ehrlich, Line integration of Ricci curvature and conjugate points in Lorentzian and Riemannian manifolds, Manuscripta Math. 31 (1980), no. 1-3, 297-316.

[3] G. J. Galloway, A generalization of Myers' theorem and an application to relativistic cosmology, J. Differential Geom. 14 (1979), no. 1, 105-116.

[4] Z. Shen, Lectures on Finsler Geometry, World Sci., 2001, Singapore. 
[5] B. Y. Wu, Volume form and its applications in Finsler geometry, Publ. Math. Debrecen 78 (2011), no. 3-4, 723-741.

[6] B. Y. Wu and Y. L. Xin, Comparison theorems in Finsler geometry and their applications, Math. Ann. 337 (2007), no. 1, 177-196.

[7] J. G. Yun, A note on the generalized Myers theorem, Bull. Korean Math. Soc. 46 (2009), no. $1,61-66$

Department of Mathematics

MinjIANG UNIVERSITY

Fuzhou 350108, Fujian, P. R. China

E-mail address: bingyewu@yahoo.cn 